\title{
A Note on the Taylor Series Expansion Coefficients of the Jacobian Elliptic Function $\operatorname{sn}(x, k)$
}

\section{By Staffan Wrigge}

\begin{abstract}
By considering $\operatorname{sn}(x, k)$ and $\operatorname{sn}^{2}(x, k)$ as power series in the modulus $k$, closed expressions are obtained for some of the integers occurring in the polynomials considered.
\end{abstract}

Introduction. During the last few years attention has been given to the problem of calculating the Taylor series expansion coefficients of the Jacobian elliptic functions $\operatorname{sn}(x, k), \operatorname{cn}(x, k)$, and $\operatorname{dn}(x, k)$. Alois Schett [4], [6] gave a combinatorial interpretation of the coefficients of $\operatorname{sn}(x, k)$ and calculated the first 25 nontrivial values of the Taylor series coefficients of $\operatorname{sn}(x, k)$ [4], [5]. Later, Dominique Dumont gave a new combinatorial interpretation of the coefficients of $\operatorname{sn}(x, k)$ and $\operatorname{cn}(x, k)$ [2]. Wrigge [8] gave recurrence formulae for the Taylor series expansion coefficients of $\operatorname{sn}(x, k)$ and $\operatorname{sn}^{2}(x, k)$ as well as closed expressions of the coefficients in terms of Legendre polynomials. In this paper we consider expansions of $\operatorname{sn}(x, k)$ and $\operatorname{sn}^{2}(x, k)$ in powers of the modulus $k$, making use of a differential equation technique to obtain closed expressions for the first two nontrivial coefficients (see Theorems I and II).

1. Definitions and Preliminaries. It is customary to consider expansions of the form

$$
\operatorname{sn}(x, k)=\sum_{n=0}^{\infty} \frac{\sigma_{2 n+1}(1, k)}{(2 n+1) !} x^{2 n+1}
$$

and

$$
\operatorname{sn}^{2}(x, k)=\sum_{n=0}^{\infty} \frac{\sigma_{2 n+2}(2, k)}{(2 n+2) !} x^{2 n+2},
$$

where $\sigma_{2 n+1}(1, k)$ and $\sigma_{2 n+2}(2, k)$ are even polynomials in the modulus $k$ of degree $2 n$. However, it is also possible to "invert" the problem, i.e., to consider expansions in powers of the modulus $k$. Thus we put

$$
\operatorname{sn}(x, k)=\sum_{n=0}^{\infty} \frac{k^{2 n}}{(2 n) !} f_{2 n}(x) ; \quad f_{0}(x)=\sin (x)
$$

and

$$
\operatorname{sn}^{2}(x, k)=\sum_{n=0}^{\infty} \frac{k^{2 n}}{(2 n) !} g_{2 n}(x) ; \quad g_{0}(x)=\sin ^{2}(x)
$$

Received July 7, 1980; revised December 5, 1980.

1980 Mathematics Subject Classification. Primary 33A25.

Key words and phrases. Elliptic functions, Laplace transforms. 
The expansions in Eqs. (1.3) and (1.4) are valid for $0 \leqslant k<1$ and $0<x<\frac{1}{2} \pi$.

2. A Differential Equation Satisfied by the Considered Functions. It is known that $\operatorname{sn}^{2}(x, k)$ satisfies the differential equation (see Bowman [1, p. 11)

$$
\frac{d^{2}}{d x^{2}} \operatorname{sn}^{2}(x, k)=2-4\left(1+k^{2}\right) \operatorname{sn}^{2}(x, k)+6 k^{2} \operatorname{sn}^{4}(x, k) .
$$

Therefore $g_{2 n}(x)$, defined by (1.4), satisfies the difference-differential equation,

$$
\begin{aligned}
\ddot{g}_{2 n}(x)= & -4 g_{2 n}(x)-8 n(2 n-1) g_{2 n-2}(x) \\
& +12 n(2 n-1) \sum_{m=0}^{n-1}\left(\begin{array}{c}
2 n-2 \\
2 m
\end{array}\right) g_{2 m}(x) g_{2 n-2-2 m}(x)
\end{aligned}
$$

with starting values $\dot{g}_{2 n}(0)=g_{2 n}(0)=0$.

Since the functions $f_{2 n}(x)$ and $g_{2 n}(x)$ are related by

$$
g_{2 n}(x)=\sum_{m=0}^{n}\left(\begin{array}{c}
2 n \\
2 m
\end{array}\right) f_{2 m}(x) f_{2 n-2 m}(x),
$$

we can, by calculating $g_{2}(x), \ldots, g_{2 n}(x)$ from (2.2) obtain expressions for $f_{2}(x), \ldots, f_{2 n}(x)$.

3. Explicit Calculations for $n=1$ and $n=2$. The differential equation satisfied by $g_{2}(x)$ is

$$
\ddot{g}_{2}(x)=-4 g_{2}(x)-8 \sin ^{2}(x)+12 \sin ^{4}(x) ; \quad g_{2}(0)=\dot{g}_{2}(0)=0 .
$$

Making use of the Laplace transform, we get

$$
g_{2}(x)=-\sin (2 x)\left[\frac{x}{2}-\frac{\sin (2 x)}{4}\right]
$$

but $g_{2}(x)=2 f_{0}(x) f_{2}(x)$, and therefore

$$
f_{2}(x)=-\cos (x)\left[\frac{x}{2}-\frac{\sin (2 x)}{4}\right]=\frac{\sin (x)}{8}+\frac{\sin (3 x)}{8}-\frac{x \cos (x)}{2}
$$

This result may also be found in Whittaker and Watson [7, p. 532]. Expanding the R.H.S. of (3.3) in powers of $x$ and comparing with (1.1), we obtain

THEOREM I. The coefficient of $k^{2}$ in the polynomial $\sigma_{2 n+1}(1, k)$ is

$$
(-1)^{n} \frac{3^{2 n+1}-3-8 n}{16}
$$

For $g_{4}(x)$ we obtain, after some manipulations, the following differential equation

$$
\begin{aligned}
\ddot{g}_{4}(x)= & -4 g_{4}(x)-12 x \sin (2 x)+18 x \sin (4 x)+3-\frac{9}{2} \cos (2 x) \\
& -3 \cos (4 x)+\frac{9}{2} \cos (6 x) .
\end{aligned}
$$

The equation is written in this form because it is then more suitable for the Laplace 
transform technique. The solution of (3.4), satisfying the required initial condition, is

$$
\begin{aligned}
g_{4}(x)= & \frac{3}{4}-\frac{3}{2} x \sin (4 x)-\frac{15}{8} x \sin (2 x)+\frac{3}{2} x^{2} \cos (2 x)+\frac{9}{64} \cos (2 x) \\
& -\frac{3}{4} \cos (4 x)-\frac{9}{64} \cos (6 x) .
\end{aligned}
$$

But from (2.3) $g_{4}(x)=6 f_{2}^{2}(x)+2 f_{0}(x) f_{4}(x)$, and therefore

$$
\begin{aligned}
f_{4}(x)= & \frac{21}{32} \sin (x)+\frac{24}{32} \sin (3 x)+\frac{3}{32} \sin (5 x)-\frac{18}{8} x \cos (x) \\
& -\frac{9}{8} x \cos (3 x)-\frac{3}{4} x^{2} \sin (x)
\end{aligned}
$$

Proceeding in the same way as before, we find

THEOREM II. The coefficient of $k^{4}$ in the polynomial $\sigma_{2 n+1}(1, k)$ is

$$
(-1)^{n} \frac{5^{2 n+1}-(8 n-4) 3^{2 n+1}+\left(32 n^{2}-32 n-17\right)}{256}
$$

This result was conjectured by my colleague Dr. Arne Fransén, who also put forward a general hypothesis for the coefficients of $k^{2 i}$ in the polynomial $\sigma_{2 n+1}(1, k) ;$ see [3].

National Defence Research Institute

Division I, Section 185

S-104 50, Stockholm 80, Sweden

1. F. Bowman, Introduction to Elliptic Functions with Applications, Dover, New York, 1961.

2. D. Dumont, "A combinatorial interpretation for the Schett recurrence on the Jacobian elliptic functions," Math. Comp., v. 33, 1979, pp. 1293-1297.

3. A. Fransén, "Conjectures on the Taylor series expansion coefficients of the Jacobian elliptic function $\operatorname{sn}(x, k)$," Math. Comp., v. 37, 1981, pp. 475-494.

4. A. SCHETT, "Properties of the Taylor series expansion coefficients of the Jacobian elliptic functions," Math. Comp., v. 30, 1976, pp. 143-147.

5. A. SCHETt, Addendum to "Properties of the Taylor series expansion coefficients of the Jacobian elliptic functions," Math. Comp., v. 31, 1977, Microfiche supplement.

6. A. SCHETt, "Recurrence formula of the Taylor series expansion coefficients of the Jacobian elliptic functions," Math. Comp., v. 31, 1977, pp. 1003-1005.

7. E. T. Whitraker \& G. N. Watson, Modern Analysis, Cambridge Univ. Press, New York, 1962.

8. S. WRIGGe, "Calculation of the Taylor series expansion coefficients of the Jacobian elliptic function $\operatorname{sn}(x, k), "$ Math. Comp., v. 36, 1981, pp. 555-564. 